\title{
DIGITAL EMULATION OF DIELECTRIC RELAXATION FUNCTIONS FOR CAPACITIVE SENSORS OF NON-DESTRUCTIVE DIELECTRIC SPECTROMETRY
}

\author{
ALDIS KALPINSH, VAIRIS SHTRAUSS \& ULDIS LOMANOVSKIS \\ Institute for Material Mechanics, University of Latvia, Latvia
}

\begin{abstract}
This paper addresses a problem of reference means for the adjusting, calibrating and testing of nondestructive dielectric spectrometers employing one-side access capacitive sensors. Due to specific features of these sensors caused by non-homogeneous electric exciting fields, the application of the conventional reference means, particularly the artificial ones, is not always possible. We propose to solve the problem by digital emulation of dielectric relaxation functions for capacitive sensors. A digital emulator is developed for one-side access capacitive sensors with active guarding for the spectrometers, working according to a two-port network measuring model. The test results are provided for digital emulation of the responses of the capacitive sensor system to sinusoidal excitations, corresponding to complex dielectric permittivity described by the Debye relaxation function, with varying of the real part of dielectric permittivity from 1 to 100 , over a frequency band from $10 \mathrm{~Hz}$ to $0.33 \mathrm{MHz}$.
\end{abstract}

Keywords: digital emulator, one-side access, capacitive sensor, dielectric spectrometer, relaxation function.

\section{INTRODUCTION}

Dielectric measurements [1] have long been of increasing interest for the characterization of non-metallic materials in many areas of science and technology, such as material science, electronics, biology, medicine, agriculture, food processing industry, etc. The potentialities of the dielectric method were significantly increased with developing one-side access (OSA) capacitive sensors [2] allowing non-destructive testing (NDT) and the characterization of materials without preparation of specimens.

Nonetheless, an actual problem for adjusting, calibrating and testing of measuring instruments of dielectric characteristics is regarding materials with the known and stable dielectric characteristics over a wide dynamic and frequency ranges. The problem is extremely urgent for dielectric spectrometers working with capacitive sensors of OSA type for reasons such as: (i) OSA sensors excite test objects by non-homogeneous electric field; (ii) the sensors have low sensing (geometric) capacitances with high stray capacitances; (iii) bulk reference specimens are needed for OSA sensors, whose minimum thickness is limited by the effective depth of penetration of electrical field; and (iv) contrary to parallel-plate capacitive sensors working with homogeneous electric field, there are no simple formulas for calculating sensing capacities of OSA sensors. The mentioned reasons do not allow or noticeably restrict to use the known solutions of artificial reference means. For example, due to small sensing capacitances of the order of hundreds and even tens femto farads, it is impossible to adjust test OSA sensors by connecting the adjustable impedances to the electrodes [3]. The limited possibilities have making artificial reference dielectrics with included electrically conductive layers [4], and producing of synthetic reference dielectrics through mixtures and liquids.

We propose to solve the problem by digital emulation of dielectric relaxation functions. Thus, the goal of this study was the development of a digital emulator for OSA capacitive 
sensors, which generates the response signals corresponding to the given dielectric relaxation function.

The rest of this work is organized in six sections. In Section 2, NDT dielectric spectrometry is considered for quality testing, in Section 3, operation of OSA capacitive sensors with active guarding is explained, the mathematical model corresponding to an equivalent circuit diagram of CSS is developed in Section 4, the implementation of the digital emulator is described in Section 5, some test results of the digital emulator are demonstrated in Section 6. Section 7 contains conclusions.

\section{NDT DIELECTRIC SPECTROMETRY FOR QUALITY TESTING}

We have developed several NDT dielectric spectrometers for solving various quality testing problems based on the ideology [2] that a lot of parameters of users' interest correlate with the dielectric characteristics, and, so many quality testing problems can be solved through NDT dielectric spectrometric measurements. Our spectrometers are classical frequency-domain instruments working over relatively low frequency band $10 \mathrm{~Hz}-1 \mathrm{MHz}$ with exciting test object with electrical field generated by sinusoidal voltage signals at discrete frequencies, usually altered geometrically with the common ratio 2 .

Two peculiar features of the spectrometers are as follows: (i) they are equipped with capacitive sensors of OSA type to accomplish non-destructive testing; and (ii) stray-immune capacitance measurements are carried out by using active guarding of the sensing electrode to increase the sensitivity and accuracy of the measurements. The capacitive sensors are protected by patents [5], [6].

A simplified representation of a NDT spectrometer is shown in Fig. 1. The spectrometer consists from three main blocks: (i) multi-frequency excitation generator; (ii) capacitive sensor system (CSS) of OSA type; and (iii) multi-frequency signal processing unit.

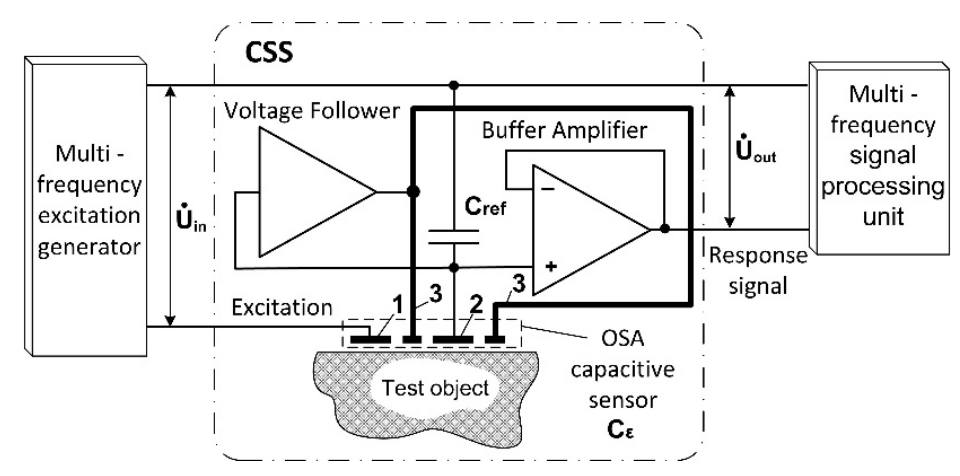

Figure 1: Simplified functional diagram of a NDT spectrometer.

\section{OSA CAPACITIVE SENSOR WITH ACTIVE GUARDING}

The capacitive sensor system (see Fig. 1) works as follows. A test object is excited by electrical field through a driven electrode 1 and a sensing electrode 2 generated by sinusoidal voltage signals from a multi-frequency excitation generator. The output signal of CSS is measured by a multi-frequency signal processing unit. A unity gain buffer amplifier is used to connect a source with a high impedance to a low-impedance load.

Both the driven electrode 1 and sensing electrode 2 are connected to the multi-frequency excitation generator, though the driven electrode 1 is connected directly, but sensing 
electrode 2 through the reference capacitor $C_{\text {ref. }}$ To carry out stray-immune capacitance measurements, the sensing electrode 2 , the reference capacitor $C_{r e f}$ and the unity gain buffer amplifier are covered with a screen, which forms a guard electrode 3 on working surface of CSS around sensing electrode. The guard electrode through a voltage follower are fed by the same voltage that of the sensing electrode. Thus, the guard electrode suppresses the electric field between the driven electrode and the sensing electrode outside the sensor working surface, so the current in the circuit of sensing electrode is made directly proportional to resultant electric field intensity in the test object. Voltage drop on the reference capacitor $C_{\text {ref }}$ created by the current in the circuit of sensing electrode forms a CSS output signal which via the buffer amplifier is passed to the multi-frequency signal processing unit.

By suppressing the electric field between the driven electrode and the sensing electrode outside the sensor working surface, the proposed solution for CSS [5] prevents the influence of stray capacitances on the output signal and ensures that the current through the sensing electrode is proportional to the capacitance between the driven electrode and the sensing electrode. We denote this capacitance as a sensing capacitance $C_{0}$ when CCS of OSA type is in the air. When the capacitive sensor is put on the test object without a gap, the sensing capacitance $C_{0}$ is multiplied by the complex dielectric permittivity of the test object

$$
\tilde{\varepsilon}(j \omega)=\varepsilon^{\prime}(\omega)-j \varepsilon^{\prime \prime}(\omega),
$$

consisting of a real part, $\varepsilon^{\prime}(\omega)$ and an imaginary part, $\varepsilon^{\prime \prime}(\omega)$, and produces a measuring capacitance

$$
\dot{C}_{\varepsilon}=C_{0} \tilde{\varepsilon}(j \omega)
$$

\section{MATHEMATICAL MODEL OF CSS}

The measuring principle of NDT dielectric spectrometers are based on the mathematical model of CSS in the form of two-port network, which an equivalent circuit diagram for analysis is demonstrated in Fig. 2.

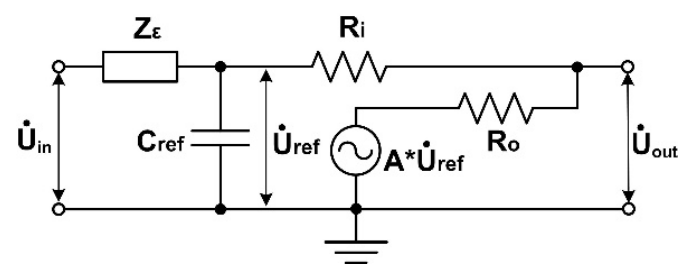

Figure 2: Equivalent circuit diagram of CSS.

The OSA capacitive sensor is represented as impedance $Z_{\varepsilon}$, while the unity gain buffer amplifier is represented by resistors $R_{i}, R o$ and voltage source $A^{*} \dot{U}_{\text {ref }}$. Due to deep negative feedback of the buffer amplifier, the input resistance $R_{i}$ is very large and the output resistance $R_{o}$ is very small. The voltage gain A of the buffer amplifier is practically equal to 1 . Therefore, it can be assumed that the output voltage $U_{\text {out }}$ practically repeats the voltage drop on the reference capacitor $C_{r e f}$ and, so $R_{i}$ and $R_{o}$ can be neglected in the mathematical model of CSS. In Fig. $2, Z_{\varepsilon}$ is the measuring (sensing) impedance

$$
Z_{\varepsilon}=\frac{1}{j \omega C_{0} \tilde{\varepsilon}(j \omega)}
$$


while $C_{\text {ref }}$ establishes a reference reactance

$$
X_{r e f}=\frac{1}{j \omega C_{r e f}} .
$$

For CSS in air,

$$
Z_{\varepsilon}=Z_{0}=\frac{1}{j \omega C_{0}} .
$$

CSS two-port network has the following transfer function

$$
K_{\varepsilon}(j \omega)=\frac{\dot{U}_{O U T}}{\dot{U}_{I N}}=\frac{X_{r e f}}{X_{r e j}+Z_{\varepsilon}},
$$

which results in

$$
K_{0}=\frac{C_{0}}{C_{0}+C_{r e f}}
$$

for CSS in air, and in

$$
K_{\varepsilon}(j \omega)=1 /\left[1+\frac{1-K_{0}}{K_{0} \tilde{\varepsilon}(j \omega)}\right]
$$

for CSS put on the test object.

Eqn (7) shows that the transfer function of two-port network for CSS in air does not depend on frequency. To measure the complex dielectric permittivity, two parameters of CSS (sensing capacitance $C_{0}$ and reference capacitance $C_{r e f}$ ) need to be known, and the amplitudes and phases of the sinusoidal input and output signals shall be measured. To emulate the dielectric relaxation functions, only $K_{0}$ must be known.

\section{IMPLEMENTATION OF DIGITAL EMULATOR}

An idea of emulation is to generate the same response signal for the given excitation signal as CSS would generate for the given complex dielectric permittivity of the test object. We are elaborated the emulator as a tool that replaces the sensor in the operations of adjusting, calibrating and testing of the spectrometer. According to eqn (8), only the transfer function for CSS in air (eqn (7)) is necessary to know to emulate the response of CSS for the given $\tilde{\varepsilon}(j \omega)$.

Therefore, for sinusoidal excitation signals, the emulator should execute three operations:

- to fix frequency for each sinusoidal excitation signal,

- to attenuate the excitation signal by a definite amount defined by a value of the complex dielectric permittivity $\tilde{\varepsilon}(j \omega)$ at the fixed frequency in the transfer function (eqn (8)), and

- $\quad$ to delay the phase of the excitation signal by a definite amount corresponding to the same value the complex dielectric permittivity.

In Fig. 3, a functional diagram is shown of a NDT spectrometer in CSS emulation mode, when CCS is replaced by the emulator. 


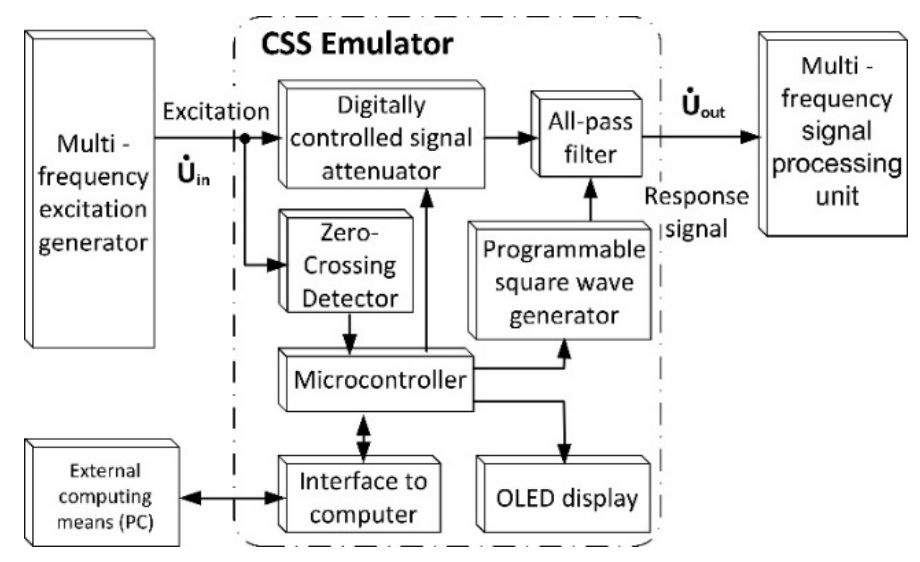

Figure 3: Functional diagram of CSS emulator.

To execute the above-mentioned operations CSS emulator includes:

- units for processing excitation signal - a digitally controlled signal attenuator and an all-pass filter,

- local computing means - a microcontroller and a OLED display;

- auxiliary control units - a programmable square wave generator, a zero-crossing detector and an interface to computer.

Excitation signal processing units generate a response signal from the given excitation signal. Local computing means controls response signal generation and displays emulator status information. The microcontroller provides the control of the operation of the signal processing units by means of auxiliary control units.

The transfer function of CSS according to the chosen dielectric relaxation function is computed by external computing means (PC) and is loaded into the microcontroller memory via the interface to computer.

\subsection{Fixing the frequency of excitation signal}

To determine the frequency, the harmonic excitation signal is converted into a periodic square waveforms by a zero-crossing detector (see Fig. 3). The duration of the period of square wave corresponds to the period of the harmonic excitation signal. The square waveforms are fed to the peripheral pin of microcontroller. The microcontroller performs measurement of the duration of the square waveform.

\subsection{Attenuation of excitation signal}

Each excitation signal at the given frequency is attenuated according to an attenuation factor determined by the complex dielectric permittivity in the transfer function (eqn (8))

$$
\left|K_{\varepsilon}(j \omega)\right|=\frac{\left|\dot{U}_{\text {OUT }}\right|}{\dot{U}_{I N}},
$$

where $\left|K_{\varepsilon}(j \omega)\right|$ indicates a part of the excitation signal to be provided at the output of the emulator. 
The proposed processing of analog signals is technically easy to implement by means of a digital-to-analog converter (DAC). In addition, the use of DAC also solves the issue of the digital control. So, a standard DAC [7] is used as a programmable divider. The circuit of the attenuator of the excitation signal is shown in Fig. 4. The digital word $D$ (control code), which determines the signal attenuation to be implemented by the DAC corresponding to the attenuation factor

$$
D=\operatorname{Int}\left\{\left|K_{\varepsilon}(j \omega)\right| \times 2^{n}+0.5\right\},
$$

is loaded to the DAC, where $n$ is the number of bits of DAC.

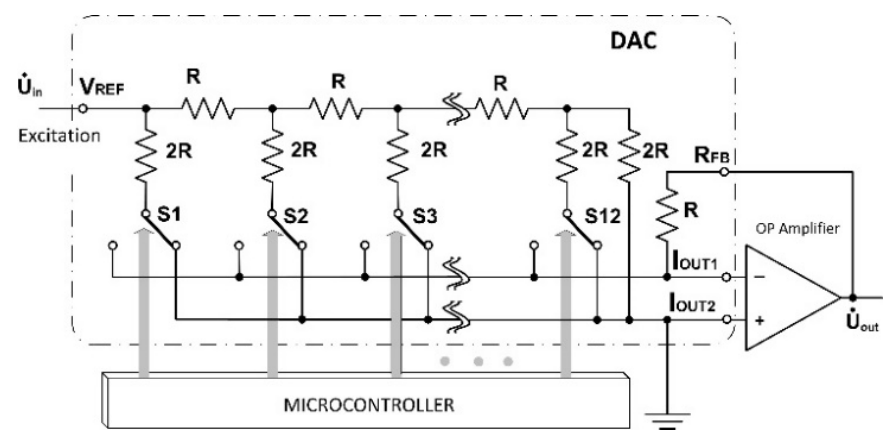

Figure 4: Circuit of digitally controlled signal attenuator.

\subsection{Delaying the excitation signal}

After attenuation of the excitation signal, phase shift of $\operatorname{Arg}\left\{K_{\varepsilon}(j \omega)\right\}$ is carried out for the signal, with no effect on the signal amplitude. Such a task is performed through a phase (all-pass) filter. For the programmable control of the procedure, a universal switched capacitor filter [8] is used. Connection diagram of filter is shown in Fig. 5.

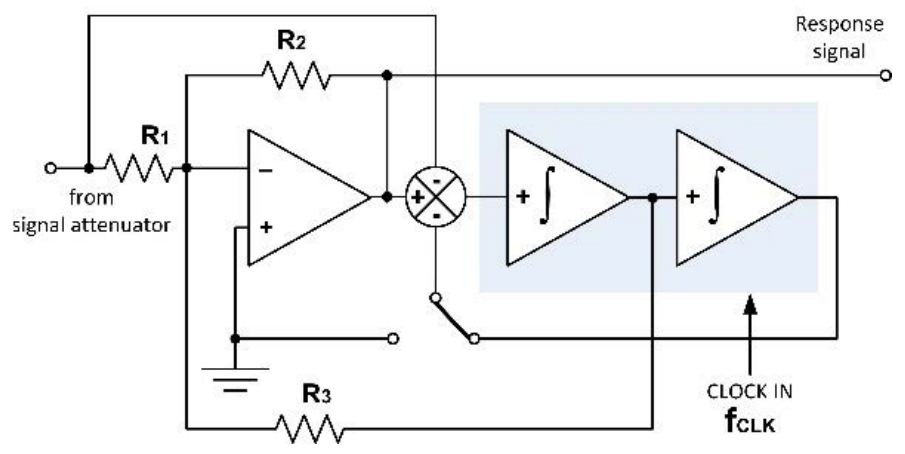

Figure 5: Block diagram of 2nd order universal switched capacitor filter.

The phase responses of all-pass filter corresponding to different center frequencies $f_{0}$ are shown in Fig. 6. It can be seen that change of the center frequency $f_{0}$ moves the phase response along the frequency axis. This means that it is possible to regulate the phase shift of signal to a specific frequency $f_{x}$ by adjusting the center frequency. 


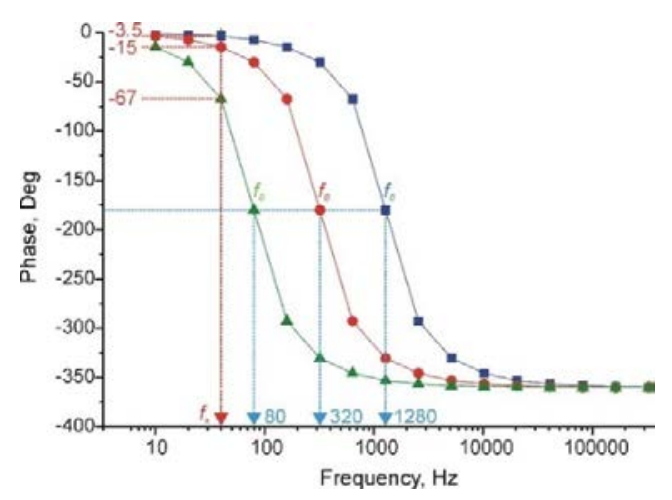

Figure 6: Phase response curves for 2-nd order all-pass filter.

The center frequency of the switched capacitor filter is set by a frequency of an external clock

$$
f_{C L K}=100 f_{0} \text {. }
$$

Thus, a microcontroller (see Fig. 3) can set a calculated phase shift $\operatorname{Arg}\left\{K_{\varepsilon}(j \omega)\right\}$ by means of a programmable square wave generator for each excitation signal.

\section{TEST RESULTS OF DIGITAL EMULATOR}

Below, the test results are provided of the digital emulator to emulate complex dielectric permittivity according to the Debye relaxation function [1]

$$
\tilde{\varepsilon}(j \omega)=\varepsilon_{\infty}+\frac{\Delta \varepsilon}{1+j \omega \tau_{0}},
$$

for $\varepsilon_{\infty}=2, \tau_{0}=10^{-3}$ and three value of $\Delta \varepsilon=1,30$ and 100 over the frequency range $10 \mathrm{~Hz}$ to $327680 \mathrm{~Hz}$ distributed geometrically with common ratio 2. The complex dielectric permittivity (eqn (12)) was emulated for OSA multi-electrode capacitive sensor with active guarding (Fig. 7) with the following technical specification.

- $\quad$ Diameter (mm)

- Minimum thickness of test object (mm) 20

- $\quad$ Sensing capacitance $(\mathrm{pF})$

- Reference capacitance $(\mathrm{pF})$

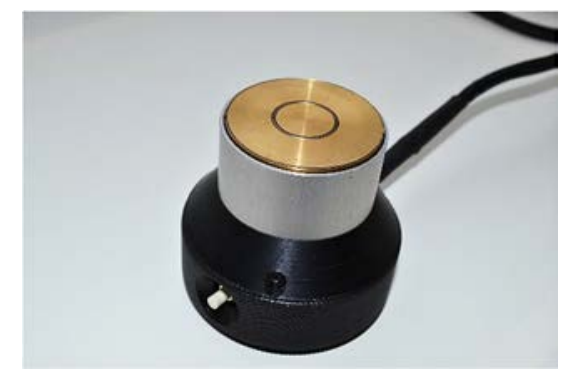

Figure 7: Photo of OSA capacitive sensor. 
In Fig. 8, relative errors are shown for the attenuation factor

$$
\delta=\frac{\left|\hat{K}_{\varepsilon}(j \omega)\right|-\left|K_{\varepsilon}(j \omega)\right|}{\left|K_{\varepsilon}(j \omega)\right|},
$$

where $\left|K_{\varepsilon}(j \omega)\right|$ is the desired attenuation factor according to eqn (9), and $\left|\hat{K}_{\varepsilon}(j \omega)\right|$ is practically measured emulated ratio of the output signal against the excitation signal.

It can be seen a tendency of increasing attenuation error with decrease of the attenuation factor. In the current implementation of the digital emulator, the relative error of signal attenuation did not exceed $1 \%$.

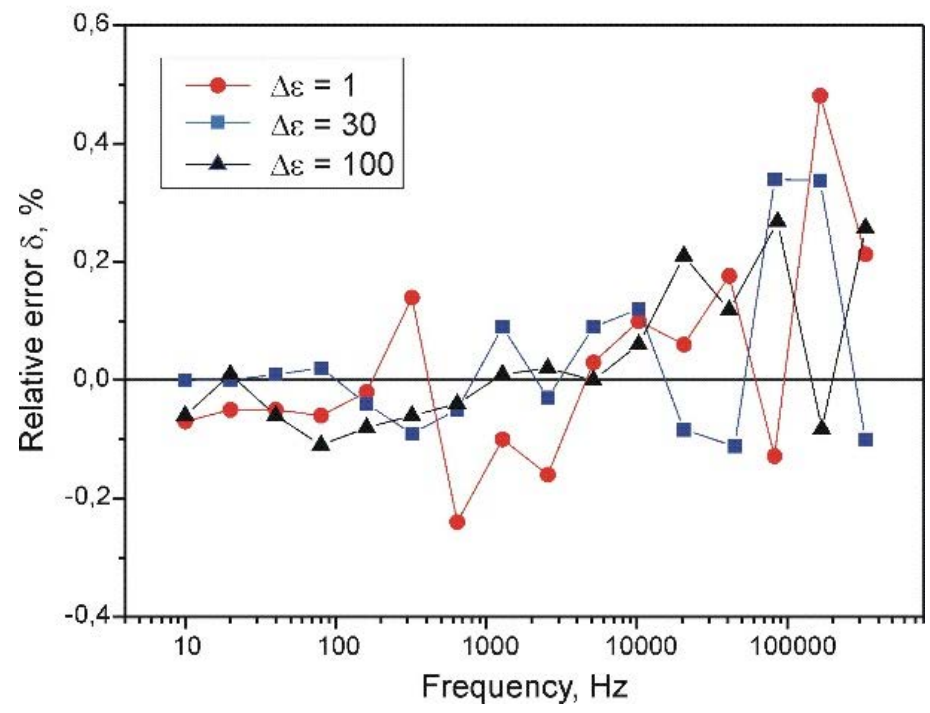

Figure 8: Relative errors of the attenuation factor.

\section{CONCLUSIONS}

We propose to solve problems of reference means for adjusting, calibrating and testing of non-destructive dielectric spectrometers employing OSA capacitive sensors by digital emulation of dielectric relaxation functions. Based on the developed two-port network measuring model of OSA capacitive sensors with active guarding working, a digital emulator has been elaborated. Tests provided for the digital emulator has witnessed its practical capability to emulate sinusoidal excitation signal attenuation with relative errors that do not exceed $1 \%$ over wide frequency band for dielectric constant varying from 1 to 100 .

\section{ACKNOWLEDGEMENTS}

This work was supported by the European Regional Development Fund under project No. 1.1.1.1/16/A/008 "Development of multi-functional tester for non-destructive quality testing of materials and structures from rigid cellular plastics".

\section{REFERENCES}

[1] Kremer, F. \& Schonhals, A., (eds) Broadband Dielectric Spectroscopy, SpringerVerlag: Berlin Heidelberg, 2003. 
[2] Shtrauss, V., Nondestructive relaxation spectrometry of dielectrics. Mech. Comp. Mat. 25(4), pp. 521-534, 1990.

[3] Boschetti, M. \& Gottardo, A., Method for calibrating apparatuses for determining the moisture content of products based on capacitive measurements, and device for stimulating the dielectric properties of products, such as wood, for use in this method. Patent document, Patent No.: US 8,410,796 B2, April 2, 2013.

[4] Troxler, R.E., Apparatuses and systems for density gauge calibration and reference emulation. Patent document, Patent No.: US 9,190,183 B2, November 17, 2015.

[5] Matiss, I., Kalpinsh, A., Shtrauss, V., Lomanovskis, U. \& Bernavs, I., Capacitance transducer for NDT of dielectric properties of materials. USSR copyright certificate, SU 1638665A1, Bulletin, 12, 1991. (In Russian.)

[6] Kalpinsh A., Shtrauss, V. \& Lomanovskis, U., Capacitive probe for non-destructive testing of dielectric materials. Patent Office of Republic of Latvia, 9, p. 1169, 2013.

[7] Analog Devices, 8-/10-/12-bit high bandwidth multiplying dacs with serial interface. Data sheet AD5426/AD/5432/AD5443. https:/www.analog.com/media/en/technicaldocumentation/data-sheets/ad5426_5432_5443.pdf.

[8] Lacanette, K., A basic introduction to filters - active, passive, and switched capacitor. National semiconductor application note 779, April 21, 2010.

www.ti.com/lit/an/snoa224a/snoa224a.pdf. 